\title{
Factors Predicting Outcomes of Tracheostomy Decannulation Among the Patients at a Tertiary Care Centre at Riyadh, Saudi Arabia - A Retrospective, Observational Study
}

\author{
Prachi Tambur, Farhan Alenezi', Winnie Philip², Sulochana Kumari³, Lojain Ebrahim Almaniei, Maram Srwi Asiri, Amjad Mubarak Alshahrani \\ Department of Respiratory Therapy, College of Applied Science, KSAU-HS, ${ }^{1}$ Consultant Critical Care, Department of Pulmonary Medicine, King AbdulAziz Medical City, \\ NGHA, ${ }^{2}$ Department of Research, College of Applied Medical Science, KSAU-HS, ${ }^{3}$ Department of Respiratory Therapy, Inaya Medical College, Riyadh, Saudi Arabia
}

\section{Abstract}

Introduction: Decannulation, the removal of the tracheostomy tube, is an essential procedure that a treating team must consider for all tracheostomized patients. There is a lack of evidence available regarding the factors that are associated with the outcomes of the decannulation process. The study aims to detect the outcome of decannulation, describe factors associated with failed decannulation and to describe the decannulation practice in adult patients at a tertiary hospital in Riyadh, Saudi Arabia. Methodology: We performed a retrospective descriptive study for 210 tracheostomized patients aged 18 and above from January 2016 to December 2017. Demographic data, tracheostomy data, and decannulation process data were collected and entered in Microsoft Excel and exported to SPSS for the analysis. Results: Among 210 patients, the majority of tracheostomies were done at the bedside (55.2\%). A total of $186(88.6 \%)$ patients were successfully decannulated within 6 months. Obesity, comorbidity, and Glasgow Coma Scale (GCS) during admission were not associated with failure. Faster decannulation (73.5\%) was found in those patients ventilated $<7$ days. Among 41 patients who underwent failed trials of decannulation, the most common reason of failure was desaturation in 19 patients $(46.3 \%)$. Six patients of them had a combination of desaturation with other factors such as secretions, low GCS, and vocal cord paralysis. Conclusion: Successful decannulation within 7 days is related to the presence of full tracheostomy team. However, having a protocol approach will be more helpful. The study showed favorable outcome for young patients and those who had an early tracheostomy in the intensive care unit.

Keywords: Decannulation protocol, safe hospital discharge, surgical tracheostomy, tracheostomy team

\section{INTRODUCTION}

Tracheostomy is an incision in the trachea done through the neck (for the insertion of a tube), to ease the passage of air for patients requiring long-term mechanical ventilation (MV) via an endotracheal tube. Tracheostomy is considered a very common procedure performed on critically ill patients. ${ }^{[1]}$ Tracheostomy has benefits such as reducing the requirement of sedation, decreasing the resistance of the airway, and increasing patient mobility. However, it can result in complications at cuff site, infection, and lower the patient's quality of life. ${ }^{[2]}$ For those mentioned reasons, the treating medical team must start the process of decannulation as soon as the medical condition of patients permits. Decannulation is the process of tracheostomy tube removal after the patient is weaned from MV and can maintain his spontaneous breathing as well as

\begin{tabular}{|l|l|}
\hline \multicolumn{2}{|c|}{ Access this article online } \\
\hline Quick Response Code: & Website: \\
\hline & www.ijrconline.org \\
\cline { 2 - 2 } & \\
\hline
\end{tabular}

protect his airway. ${ }^{[3]}$ The decannulation process has many advantages such as improving swallowing function, physical appearance and patient comfort. ${ }^{[4]}$

The practice of decannulation is not standardized, and there is no accepted universal protocol for this transitional process. This lack of evidence can lead to a variation of practice between

Address for correspondence: Ms. Sulochana Kumari, Department of Respiratory Therapy, Inaya Medical College, Building No-10, P. 0. Box 271 880, Riyadh 11352, Saudi Arabia. E-mail: Sulochana.rt@gmail.com

This is an open access journal, and articles are distributed under the terms of the Creative Commons Attribution-NonCommercial-ShareAlike 4.0 License, which allows others to remix, tweak, and build upon the work non-commercially, as long as appropriate credit is given and the new creations are licensed under the identical terms.

For reprints contact: reprints@medknow.com

How to cite this article: Tambur P, Alenezi F, Philip W, Kumari S, Almaniei LE, Asiri MS, et al. Factors predicting outcomes of tracheostomy decannulation among the patients at a tertiary care centre at Riyadh, Saudi Arabia - A retrospective, observational study. Indian J Respir Care 2020;9:199-203.

Received: $29-01-2020$

Accepted: $30-03-2020$

Revised: 09-02-2020 Published: 19-06-2020 
clinicians in the decision for decannulation and prolonged need for MV. Furthermore, the level of consciousness, cough effectiveness, and amount of secretions are often not the maximum requirements for successful decannulation. This tertiary hospital has a multidisciplinary team for tracheostomized patients that include a nurse, respiratory therapist, ENT surgeon, speech therapist, and a coordinator. Each of them does a daily round on all tracheostomized patients. This team has many tasks including changing tracheostomy tubes, dealing with secretions, educating the family and initiation of the decannulation process. We think that the presence of a protocolized approach can help the treating medical team in better judgment, know expectations from the procedure, and prepare the patients and their families.

There is a lack of studies on the factors predicting the outcome of decannulation in Saudi Arabia. This study was aimed to look at factors that predict the success of decannulation in the presence of the multidisciplinary team and a protocolized approach to tracheostomized patients.

\section{Methodology}

This study was a cross-sectional, retrospective database review at this tertiary care center at Riyadh for all tracheostomized patients aged 18 years and above who were admitted to the intensive care units (ICUs) and wards over 2 years (from January 2016 to December 2017). Patients with Guillain-Barre syndrome, patients on chronic ventilation, and 'No Code' patients were excluded from the study. Data from 210 eligible patients were collected. This study was approved by the institutional review board.

The data were collected using a data collection sheet. The baseline demographic variables, which include gender, age, body mass index (BMI), admission diagnosis, comorbidity and Glasgow Coma Scale (GCS) during admission were collected. Data regarding tracheostomy included the duration of tracheostomy, method of tracheostomy, whether surgical or percutaneous, tracheostomy tube type and size, complication during the first $48 \mathrm{~h}$ were also noted. Type and duration of MV were also recorded. Data that describe the corking process, outcome of decannulation, and the reasons for failed trials of decannulation, any accidental removal of tracheostomy tube, GCS and amount of secretions before and after decannulation and finally the patient's dispositions were studied. The participants were classified into two groups based on age group, $\leq 50$ years and $>50$ years. They were also categorized based on BMI into underweight, normal, obese, and overweight groups. The admission diagnosis was distributed into main groups (cancer, respiratory problems and diseases, infectious diseases, neurological diseases, cardiac diseases, burn, trauma, motor vehicle accident [MVA], and others). The comorbidities were also classified into chief groups (hypertension, diabetes, central nervous system, liver, heart, kidney, and respiratory diseases). The duration of tracheostomy was categorized into three groups
( $\leq 6$ months and $>6$ months, still on tracheostomy). The type of ventilation was classified into four types, including MV, automatic tube compensation (ATC), humidified air, and room air. We classified the duration of MV into three groups, patients who were ventilated for $\leq 7$ days, from 8 to 30 days, and for $>30$ days. Similarly, the duration of corking was categorized in $\leq 7$ days and $>7$ days. The outcome of decannulation was evaluated based on whether the removal of the tracheostomy tube attempt succeeded or failed. It was classified into four categories: patients who had successful decannulation with 1 trial, patients who had successful decannulation with $>1$ trial, patients who had failed decannulation with 1 trial, and patients who had failed decannulation with $>1$ trial. In patients' dispositions, the classification was as follows: (death, went home, transferred into another hospital, sent to the long-term care facility, and continuing in the hospital).

The collected data were entered in MS Excel and transferred to SPSS version 22 Clearthought Software License v2.0 Riyadh Saudi Arabia for further statistical analysis. Tables and figures were used to represent the result. Frequency and percentage were used for categorical variables. Continuous variables were represented as mean and standard deviation. Pearson's Chi-square and independent $t$-test were used in the study. The relationship between the outcome of the study and categorical variables were tested using Chi-square, whereas those with continuous variables were tested using independent $t$-test. $P<0.05$ was considered to be statistically significant.

\section{RESULTS}

A total of 210 patients who were 18 years of age or older included in the study. Most of the patients were men (78.1\%) with a mean age of 45.75 years and normal BMI $(35.2 \%)$. The most common cause for ICU admission was MVA (40.5\%). They presented with very low GCS (48.1\%). One hundred and six patients presented with comorbidities. Majority of patients had hypertension $(66 \%)$ followed by diabetes mellitus $(56.6 \%)$. A few patients had coexisting hepatic disorders $(9.4 \%)$. The baseline characteristics of these 210 patients are shown in Table 1.

Among 210 patients who underwent decannulation process, 186 patients $(88.6 \%)$ had successful decannulation and a majority of them $(79.58 \%)$ succeeded in the first trial [Figure 1]. Failure of decannulation was reported in 24 patients $(11.4 \%)$. Of them, $9.01 \%$ had failed decannulation from the first trial, and $2.40 \%$ with two trials or more. The reasons for failed decannulation trials were desaturation, low GCS, secretions, vomiting, tachycardia, stridor, weak cough reflex, vocal cord paralysis, respiratory insufficiency, epilepsy, and tachypnea. The most common cause was desaturation (46.3\%). Five patients had a combination of desaturation with other factors such as secretions, low GCS, and vocal cord paralysis. Successfully decannulated patients had very less secretion before and after decannulation with GCS $>12$.

Successfully decannulated patients were predominantly men $(80.6 \%)$, aged $<50$ years $(62.4 \%)$ with normal body 


\begin{tabular}{lc}
\hline Table 1: Basic characteristics of the study subjects \\
\hline Variable & $\boldsymbol{n}(\%)$ \\
\hline Gender & \\
Male & $164(78.1)$ \\
Female & $46(21.9)$ \\
Age in years & \\
S50 & $123(58.6)$ \\
>50 & $87(41.4)$ \\
BMI (kg/m $\left.{ }^{2}\right)$ & \\
Underweight & $16(7.6)$ \\
Normal weight & $74(35.2)$ \\
Obese & $58(27.6)$ \\
Overweight & $62(29.5)$ \\
Admission diagnosis & \\
Cancer & $11(5.2)$ \\
Respiratory diseases and problems & $22(10.4)$ \\
Infectious diseases & $11(5.2)$ \\
Neurological diseases & $34(16.1)$ \\
Motor vehicle accidents & $85(40.5)$ \\
Burn & $13(6.2)$ \\
Cardiac problems & $11(5.2)$ \\
Others & $23(11)$ \\
Comorbidities & \\
Hypertension & $70(66)$ \\
Diabetes mellitus & $60(56.6)$ \\
CNS & $22(20.8)$ \\
Liver & $43(40.6)$ \\
Heart & $24(22.6)$ \\
Kidney & $32(30.18)$ \\
Respiratory & $106(50.47)$ \\
GCS during admission & \\
Mild 13-15 & $62(29.5)$ \\
Moderate 9-12 & $47(22.4)$ \\
Severe 3-8 & $101(48.1)$ \\
\hline mass index \\
\end{tabular}

weight (36\%). There was no significant association between BMI, GCS during admission and failure of decannulation [Table 2].

There was no significant association between comorbidities and outcome of decannulation [Figure 2].

Patients who had tracheostomy consequent to motor vehicle accidents had maximum successful decannulation rate as compared to those with a neurological disease such as stroke or intracranial hemorrhage [Figure 3].

Table 3 shows no significant association between tracheostomy method and outcome of decannulation $(P=0.606)$. The majority of tracheostomies were done bedside $(55.2 \%)$ and most of them (88.6\%) were successfully decannulated within 6 months. The overall complication observed within $48 \mathrm{~h}$ after the tracheostomy procedure was minimal bleeding which is normal. The accidental removal of the tracheostomy tube was recorded in $3.8 \%$ of subjects but were subsequently successfully decannulated. Faster decannulation was observed

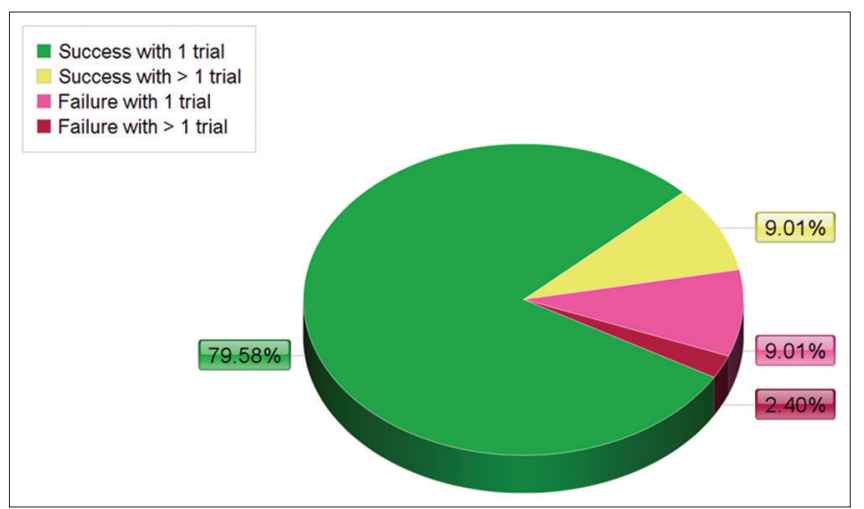

Figure 1: Outcome of decannulation

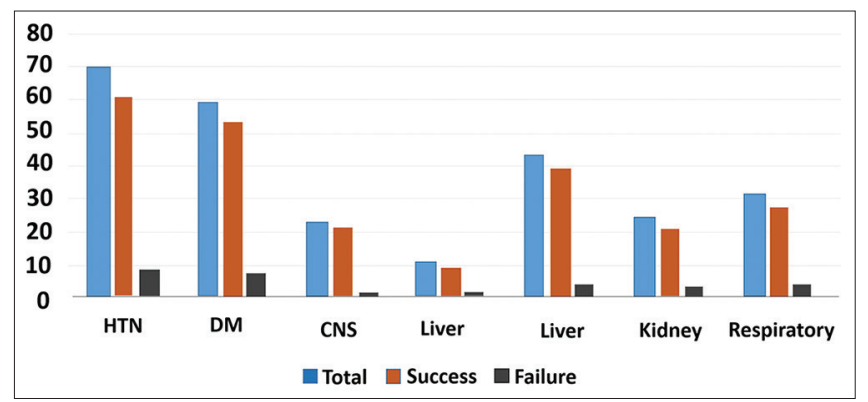

Figure 2: Association between comorbidities and outcome of decannulation

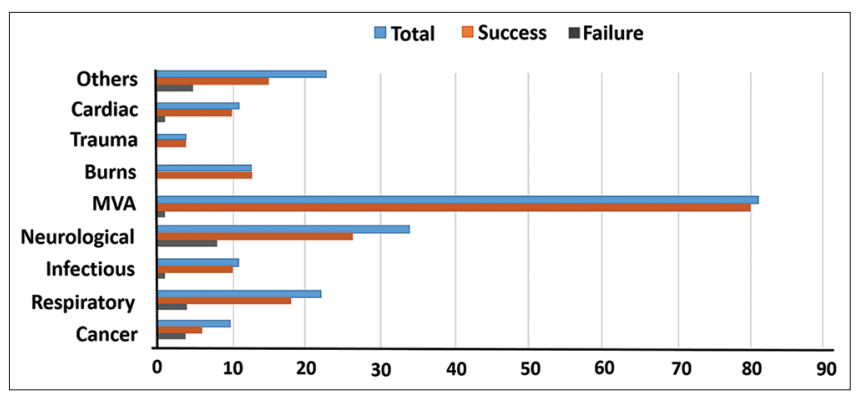

Figure 3: Admission diagnosis and success of tracheostomy decannulation

in patients $(73.5 \%)$ who required mechanical ventilatory support for $<7$ days.

\section{Discussion}

The practice of decannulation is not standardized, and there is no accepted universal protocol for this transitional process. This tertiary hospital has a multidisciplinary team for tracheostomized patients. This study was aimed to look at factors that predict the success of decannulation in the presence of multidisciplinary team and a protocolized approach to tracheostomized patients.

A cross-sectional study done in 2008 at 118 medical centers of London investigating decannulation practice found that level of consciousness, secretions, and oxygenation are the most 
Tambur, et al:: Factors predicting decannulation outcomes

\begin{tabular}{|c|c|c|c|}
\hline Variable & $\begin{array}{c}\text { Success } \\
(n=168), n(\%)\end{array}$ & $\begin{array}{c}\text { Failed } \\
(n=24), n(\%)\end{array}$ & $P$ \\
\hline \multicolumn{4}{|l|}{ Age (years) } \\
\hline$\leq 50$ & $116(62.4)$ & $7(29.2)$ & 0.0020 \\
\hline$>50$ & $70(37.6)$ & $17(70.8)$ & \\
\hline \multicolumn{4}{|l|}{ Gender } \\
\hline Male & $150(80.6)$ & $14(58.3)$ & 0.013 \\
\hline Female & $36(19.4)$ & $10(41.7)$ & \\
\hline \multicolumn{4}{|l|}{ BMI $\left(\mathrm{kg} / \mathrm{m}^{2}\right)$} \\
\hline Under weight & $13(7.0)$ & $3(12.5)$ & 0.651 \\
\hline Normal weight & $67(36.0)$ & $7(29.2)$ & \\
\hline Obese & $50(26.9)$ & $8(33.3)$ & \\
\hline Overweight & $56(30.1)$ & $6(25.0)$ & \\
\hline \multicolumn{4}{|c|}{ GCS during admission } \\
\hline Mild 13-15 & $55(29.6)$ & $7(29.2)$ & 0.9450 \\
\hline Moderate 9-12 & $41(22)$ & $6(25.0)$ & \\
\hline Severe 3-8 & $90(48.4)$ & $11(45.8)$ & \\
\hline
\end{tabular}

GCS: Glasgow Coma Scale, BMI: Body mass index

\begin{tabular}{|c|c|c|c|}
\hline \multirow[t]{2}{*}{ Variable } & \multicolumn{2}{|c|}{ Decannulation outcome } & \multirow[t]{2}{*}{$P$} \\
\hline & $\begin{array}{c}\text { Success } \\
(n=186), n(\%)\end{array}$ & $\begin{array}{c}\text { Failed }(n=24), \\
n(\%)\end{array}$ & \\
\hline \multicolumn{4}{|c|}{ Tracheostomy method } \\
\hline Surgical & $67(36)$ & $11(45.8)$ & 0.606 \\
\hline Percutaneous & $105(56.5)$ & $11(45.8)$ & \\
\hline Unknown & $14(7.5)$ & $2(8.3)$ & \\
\hline \multicolumn{4}{|c|}{$\begin{array}{l}\text { Tracheostomy duration } \\
\text { (months) }\end{array}$} \\
\hline$\leq 6$ & $176(94.6)$ & $10(83.3)$ & 0.112 \\
\hline$>6$ & $10(5.4)$ & $2(16.7)$ & \\
\hline Unknown & 0 & 12 & \\
\hline \multicolumn{4}{|l|}{$\begin{array}{l}\text { Duration of corking } \\
\text { (days) }\end{array}$} \\
\hline$\leq 7$ & $175(94.1)$ & $12(50)$ & 0.001 \\
\hline$>7$ & $11(5.9)$ & $1(4.2)$ & \\
\hline Unknown & 0 & $11(45.8)$ & \\
\hline \multicolumn{4}{|l|}{ Types of ventilation } \\
\hline MV & 147 (79) & $19(79.2)$ & 0.454 \\
\hline ATC & $25(13.4)$ & $5(20.8)$ & \\
\hline Humidified gases & $5(2.7)$ & 0 & \\
\hline Room air & $9(4.8)$ & 0 & \\
\hline \multicolumn{4}{|c|}{ Duration of MV (days) } \\
\hline$\leq 7$ & $108(73.5)$ & $14(73.7)$ & 0.812 \\
\hline $8-30$ & $35(23.8)$ & $4(21.1)$ & \\
\hline$>30$ & $4(2.7)$ & $1(5.3)$ & \\
\hline Total & 147 & 19 & \\
\hline
\end{tabular}

important determinants of clinician's decision to decannulate. ${ }^{[5]}$ A retrospective study of 135 patients, performed in 2009 in long-term facility ${ }^{[6]}$ reported that decannulation was achieved in $35 \%$ of the patients who had $<45$ days of tracheostomy insertion. Patients who failed decannulation trial had a tracheostomy tube placed earlier and had shorter ICU length of stay as compared with patients who were decannulated successfully.

According to a study conducted in regional weaning centre at Donaustauf Hospital, Germany, 2012, where they studied tracheal retainer as the protocolized approach found out that low oxygenation, delayed period of spontaneous breathing, age and high Simplified Acute Physiology Score are best detectors for the prolonged need of MV and decannulation failure. ${ }^{[7]}$

A retrospective study from patients' records published in 2017, identified the presence of a spontaneous cough and mean expiratory pressure as the essential factors to predict the success of decannulation, while GCS has no effect on the outcome of tube removal which was supported with other studies. ${ }^{[8,9-12]}$ Other studies considered the absence of psychiatric illnesses and appropriate level of consciousness ${ }^{[13-15]}$ as essential factors in the decannulation process.

A systematic review published in 2014 reported that protocol for evaluation of swallowing assessment and dysphagia as part of a structured team can play a major role in the decannulation process. ${ }^{[16]}$ It was also suggested that the presence of a clear protocol and multidisciplinary team can increase the chances of successful decannulation. ${ }^{[17]}$

Most of the patients in this study were males with a mean age of 45.75 years. The majority of tracheostomy procedures performed in this hospital were done percutaneously $(55.2 \%)$ at bedside by intensivist or ENT surgeons and almost $88.6 \%$ of these patients got successfully decannulated within 6 months. Bleeding was the only complication that was noted during the tracheostomy procedure. Performance of bedside percutaneous tracheostomy procedure shows lower bleeding risk as compared to surgical procedure.

Factors such as obesity, presence of comorbidities, and GCS during admission were not associated with failure. It was also observed that during ICU admission, factors such as age can show significant association with decannulation success rate. In this study, elderly patients ( $>50$ years) with comorbidities showed higher risk of decannulation failure than younger patients ( $<50$ years).

Good neurological status of a patient is important for early weaning from MV before decannulation. Faster and successful decannulation (73.5\%) was achieved in those patients who required less MV support, especially $<7$ days and in those who had a higher GCS on discharge from ICU. The GCS level at hospital discharge influences decannulation outcome, which was an insignificant factor in previous studies. ${ }^{[5,8-12]}$

Previous studies have shown that presence of factors such as desaturation episodes, poor neurological responses, hemodynamic instability, vocal cord dysfunction etc., can lead to poor prognosis for successful decannulation. In this study, of the 24 patients who failed decannulation trials we found 
Tambur, et al:: Factors predicting decannulation outcomes

that almost 19 patients (46.3\%) had desaturation episodes only during the decannulation trial. The remaining five patients had a combination of desaturation episodes along with other factors such as secretions, low GCS, and vocal cord paralysis.

The fractional inspired concentration of oxygen required $\left(\mathrm{FIO}_{2}\right)$ was also demonstrated as an essential factor in decannulation outcome, along with the amount of secretions, which is consistent with previous studies. Furthermore, lower duration of tracheostomy was associated significantly with successful decannulation $(88.6 \%){ }^{[6]}$ We found out that decannulation practice with the presence of multidisciplinary team, including respiratory therapist can help in faster decannulation.

This study was limited by its retrospective design, secondary data being often prone to nonprecision and less-reporting information. Further prospective and multicenter trials are recommended.

\section{Conclusion}

Successful decannulation is likely in tracheostomized patients when the patients are younger, if they have had an early tracheostomy and those with a lesser amount of secretions. The presence of full multidisciplinary tracheostomy team would increase the chance of decannulation and help the family when dealing with their patient. We recommend having a protocol approach and clear communication with the treating team for better outcomes.

\section{Financial support and sponsorship}

Nil.

\section{Conflicts of interest}

There are no conflicts of interest.

\section{References}

1. Krishnan K, Elliot SC, Mallick A. The current practice of tracheostomy in the United Kingdom: A postal survey. Anaesthesia 2005;60:360-4.

2. Engels PT, Bagshaw SM, Meier M, Brindley PG. Tracheostomy: From insertion to decannulation. Cancer J Surg 2009;52:427-33.

3. Kutsukutsa J, Mashamba-Thompson TP, Saman Y. Tracheostomy decannulation methods and procedures in adults: A systematic scoping review protocol. Syst Rev 2017;6:4-9.

4. O 'Connor HH, White AC. Tracheostomy decannulation introduction process of weaning and routine decannulation managing accidental decannulation postdecannulation monitoring decannulation failure and alternatives to decannulation summary. Respir Care 2010;55:1076-81.

5. Stelfox HT, Crimi C, Berra L, Noto A, Schmidt U, Bigatello LM, et al. Determinants of tracheostomy decannulation: An international survey. Crit Care 2008;12:1-9.

6. O'Connor HH, Kirby KJ, Terrin N, Hill NS, White AC. Decannulation following tracheostomy for prolonged mechanical ventilation. J Intensive Care Med 2009;24:187-94.

7. Budweiser S, Baur T, Jörres RA, Kollert F, Pfeifer M, Heinemann F. Predictors of successful decannulation using a tracheostomy retainer in patients with prolonged weaning and persisting respiratory failure. Respiration 2012;84:469-76.

8. Perin C, Meroni R, Rega V, Braghetto G, Cerri C. Parameters influencing tracheostomy decannulation in patients undergoing rehabilitation after severe acquired brain injury: Parameters influencing tracheostomy decannulation in patients undergoing rehabilitation after severe acquired brain injury (SABI). Int Arch Otorhinolaryngol 2017;21:382-9.

9. Hernández G, Ortiz R, Pedrosa A, Cuena R, Vaquero Collado C, González Arenas $\mathrm{P}$, et al. The indication of tracheotomy conditions the predictors of time to decannulation in critical patients. Med Intensiva (English Ed) 2012;36:531-9. Available from: http://www. elsevier.es/medintensiva. [Last accessed on 2012 Nov 25].

10. Santus P, Gramegna A, Radovanovic D, Raccanelli R, Valenti V, Rabbiosi $\mathrm{D}$, et al. A systematic review on tracheostomy decannulation: A proposal of a quantitative semiquantitative clinical score. BMC Pulm Med 2014;14:1-8.

11. Duan J, Liu J, Xiao M, Yang X, Wu J, Zhou L. Voluntary is better than involuntary cough peak flow for predicting re-intubation after scheduled extubation in cooperative subjects. Respir Care 2014;59:1643-51. Available from: http://rc.rcjournal.com/cgi/doi/10.4187/respcare. 03045 .

12. Ahmed R, Tabish S, Zaidi R, Husein H, Moshtohry H, Ahmad K, et al. Early decannulation, repatriation, and hospital discharges by introducing a tracheostomy care task force for noncritical care tracheostomized patients. Ashdin Public J Orthop Trauma 2012;2:4.

13. Ceriana P, Carlucci A, Navalesi P, Rampulla C, Delmastro M, Piaggi G, et al. Weaning from tracheotomy in long-term mechanically ventilated patients: Feasibility of a decisional flowchart and clinical outcome. Intensive Care Med 2003;29:845-8.

14. Christopher KL. Tracheostomy decannulation. Respir Care 2005;50:538-41.

15. De Leyn P, Bedert L, Delcroix M, Depuydt P, Lauwers G, Sokolov Y, et al. Tracheotomy: Clinical review and guidelines. Eur J Cardiothorac Surg 2007;32:412-21.

16. Garuti G, Reverberi C, Briganti A, Massobrio M, Lombardi F, Lusuardi M. Swallowing disorders in tracheostomised patients: A multidisciplinary/multiprofessional approach in decannulation protocols. Multidiscip Respir Med 2014;9:1-10.

17. Bertolino C, Biacca M, Mazzari P, Comberiati R, Giordano D, Mezzadri E, et al. Integrated management protocol of the tracheostomy tube in severe brain injury: Preliminary results. In: SIMFER, editor. Proceedings Abstract Book of the $37^{\text {th }}$ National Congress of Italian Society of Physical and Rehabilitation Medicine: 20-23 September 2009. Campobasso; 2009. p. 53. 